\title{
Non-Periodic Epsilon-Near-Zero Metamaterials at Visible Wavelengths for Efficient Non-Resonant Optical Sensing
}

Zelio Fusco ${ }^{\mathrm{a}}$, Mahdiar Taheri ${ }^{\mathrm{b}}$, Renheng Bo ${ }^{\mathrm{a}}$, Thanh Tran-Phü, Hongjun Chen ${ }^{\mathrm{a}}$, Xuyun Guo ${ }^{\mathrm{c}}, \mathrm{Ye}$ $Z_{\text {Zhu }}$, Takuya Tsuzuki ${ }^{b}$, Thomas P. Whited ${ }^{\mathrm{d}}$, and Antonio Tricoli ${ }^{\mathrm{a}}$

a Nanotechnology Research Laboratory, College of Engineering and Computer Science, The Australian National University, ACT 2601, Australia

${ }^{\mathrm{b}}$ Laboratory of Advanced Nanomaterials for Sustainability, College of Engineering and Computer Science, The Australian National University, ACT 2601, Australia

c Department of Applied Physics, The Hong Kong Polytechnic University, Hong Kong

d Research School of Electrical, Energy and Materials Engineering, The Australian National University, ACT 2601, Australia 


\title{
Flame Spray Pyrolysis
}

\author{
$\mathrm{Na}_{\mathrm{x}} \mathrm{WO}_{3}$ \\ Deposition \\ !
$\vdots$
Coagulation \\ Condensation \\ Nucleation \\ Evapóration \\ Atomization
}
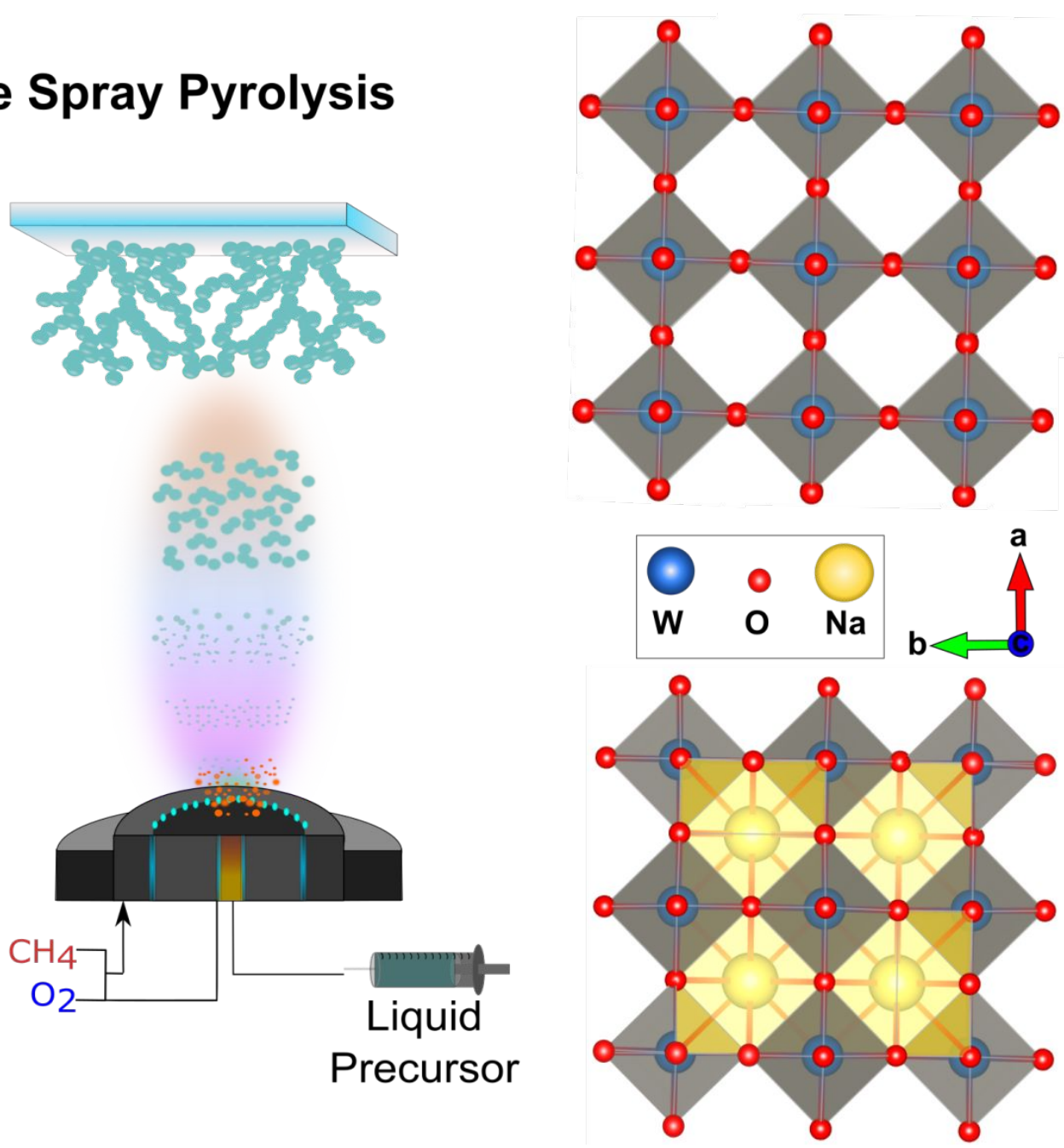

Figure S1. Schematic representation of the flame spray pyrolysis (left) and the main physical processes that are happening within the luminous region of the flame. We synthesize a continuous aerosol of Na-doped $\mathrm{WO}_{3}$ nanoparticles (with atomic $\mathrm{Na} / \mathrm{W}$ ratio from 0 to 1 ) by flame spray combustion of a precursor solution of sodium acetate and ammonium (meta)tungstate hydrate in xylene, with atomic $\mathrm{Na} / \mathrm{W}$ ratio from 0 to 1 ). We exploit the thermphoretic gradient between the hot aerosol and a cooled substrate to direct the aerosol nanoparticles toward targeted surfaces, such as quartz substres, leading to the self-assembly of networks of $\mathrm{Na}$ doped $\mathrm{WO}_{3}$ nanoparticles. A deposition rate of $\sim 2.5 \mu \mathrm{m} / \mathrm{min}$ of the precursor solution was obtained over surfaces of more than $2 \mathrm{~cm}^{2}$ this is in line with previous report on the direct aerosol deposition of doped ${ }^{1}$ and pure $^{2}$ metal oxide nanoparticles by flame spray pyrolysis. The $\mathrm{Na}$ doped $\mathrm{WO}_{3}$ nanoparticle network morphology is independent of the sodium content, and characterized by a very high porosity in the range of $98 \%$ (Figure 1c). These structural properties are in line with those previously reported for aerosol selfassembly of nanoparticles in the diffusion-limited regime ${ }^{3-5}$. The first step of the flame spray pyrolysis process involves the atomization of the precursor solution in fine droplets that are than exposed to the luminous region of the flame $(>2000 \mathrm{~K})$. At these temperatures the organic components of the precursor and solvent solution are oxidated to $\mathrm{CO}_{2}$ and $\mathrm{H}_{2} \mathrm{O}$. Some carbon compounds may still be able to provide trace contaminants on the particle surface. The composition of the synthesized $\mathrm{Na}_{\mathrm{x}} \mathrm{WO}_{3}$ has been analized by XRD, (Figure 3b), and, 
despite the presence of minor contaminants cannot be excluded, no other crystalline species were identified.

Further thermal annealing in reducing atmosphere $\left(5 \% \mathrm{H}_{2}\right.$ in $\left.\mathrm{N}_{2}\right)$, which promotes the sodium intercalation in the $\mathrm{WO}_{3}$ lattice, is needed for the successful synthesis of $\mathrm{Na}_{\mathrm{x}} \mathrm{WO}_{3}$. The high temperature and the reducing atmosphere start to induce a morphological change which progressively leads to lower porosity and formation of regular nanostructured cubes. The purity of our synthesized $\mathrm{Na}_{\mathrm{x}} \mathrm{WO}_{3}$ has been analized by meaning of $\mathrm{XRD}$, see Figure $3 \mathrm{~b}$ in the main text, and, despite we can't exclude the presence of trace contaminants, we confirm that no other chemical species having a crystalline nature are present on our samples.

Crystal structures of $\mathrm{WO}_{3}$ (top-right) and $\mathrm{NaWO}_{3}$ (bottom-right). The most common structure of $\mathrm{WO}_{3}$ at room temperature is monoclinic whose three-dimensional structure consist of $\mathrm{WO}_{6}$ octahedral with oxygen atoms placed at each corner. With the increase of intercalated $\mathrm{Na}$, the compound becomes metallic and shows a cubic symmetry with the $\mathrm{Na}$ ions displaced at the centre of the cube, while the $\mathrm{W}$ atoms are located at the corners. When this architecture is periodically repeated within the lattice, each $\mathrm{W}$ forms an octahedral framework with $6 \mathrm{O}$ $\left(\mathrm{WO}_{6}\right)$ located at the corner of each cubic single cell. At lower $\mathrm{Na}$ fractions $(\mathrm{x}<0.25)$, the intrinsic disorder and Anderson localization lead to long-range Coulombic interactions which restrict free electron movement, resulting in semiconductor-like properties. ${ }^{6}$ Above the threshold, further doping of $\mathrm{Na}$ ions causes the delocalization of the electron states and the system undergoes a metal-insulator transition, which can be well explained by a rigid shift of the band structure ${ }^{6}$. 
S2 - Scanning Transmission Electron Microscopy
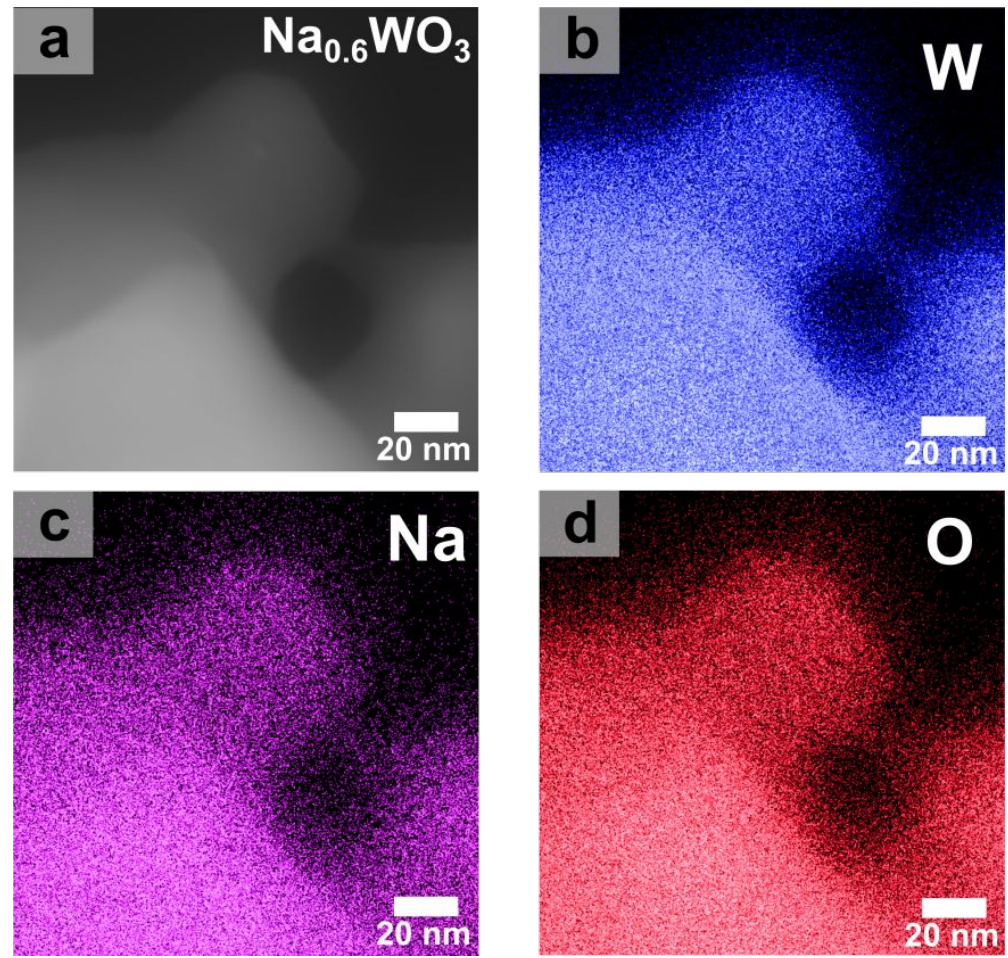

Figure S2. a) Scanning transmission electron micrographs of a $\mathrm{Na}_{0.6} \mathrm{WO}_{3}$ crystals and (b-d) elemental mapping of tungsten, sodium and oxygen. The elemental analysis demonstrates that the sodium is uniformly intercalated through the whole crystal. 


\section{S3 - Transmission Electron Microscopy}

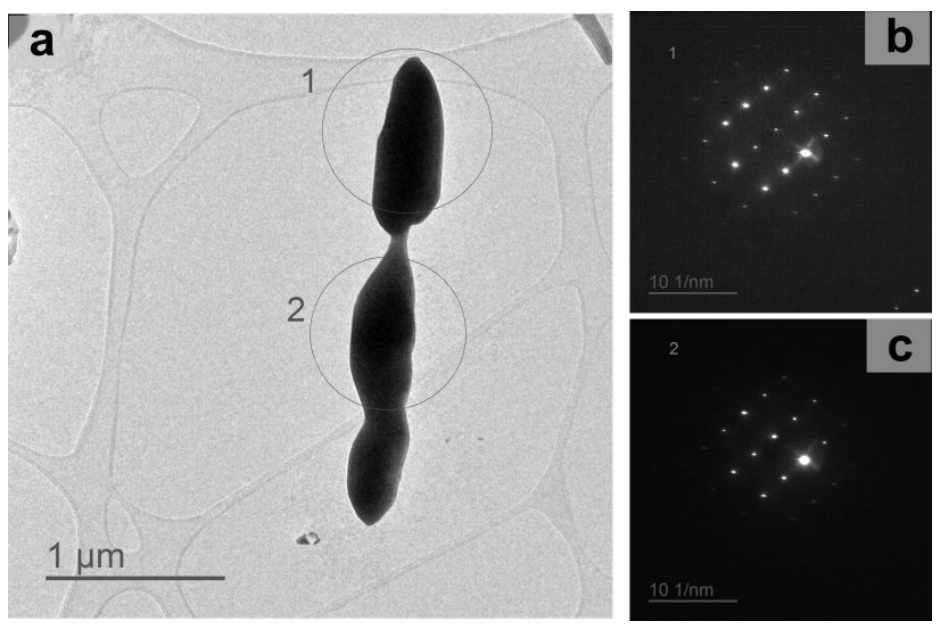

Figure S3. a) Exemplar transmission electron micrograph of a needle-like $\mathrm{Na}_{0.6} \mathrm{WO}_{3}$ structure. (b-c) Selected area electron diffraction patterns (SAED) from the two regions highlighted in panel a, showing the same diffraction spots meaning that the structure is a single crystal. 


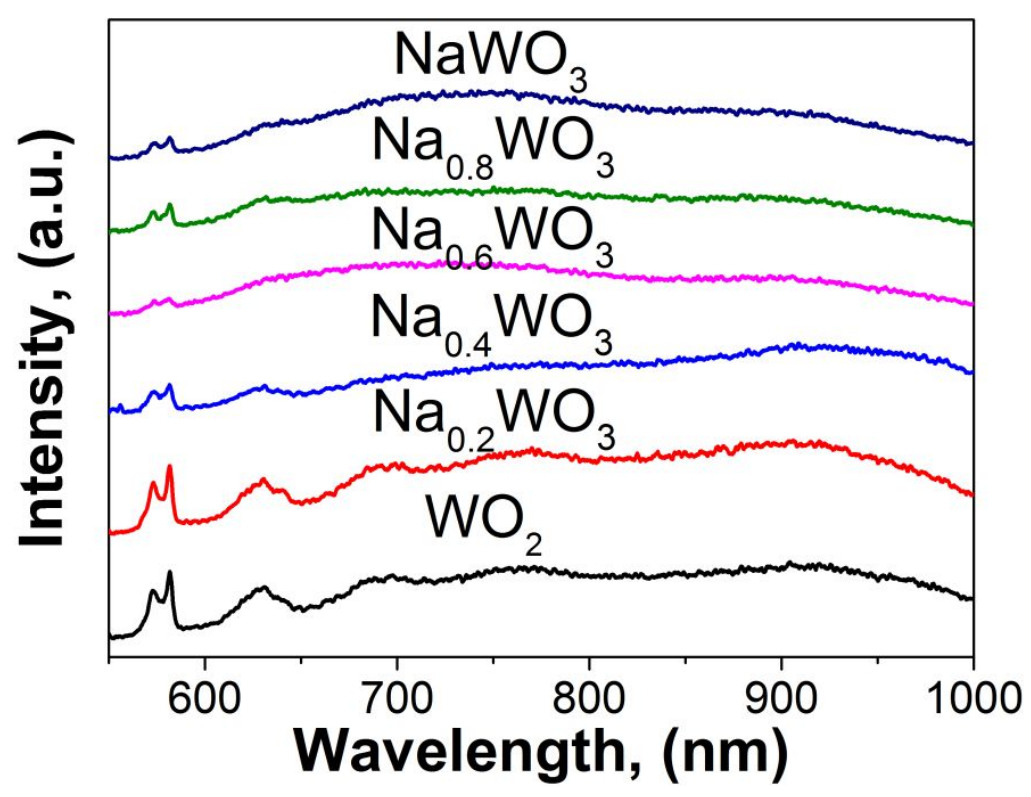

Figure S4. Stacked photoluminescence spectra for the different $\mathrm{Na}_{\mathrm{x}} \mathrm{WO}_{3}$ with $0<\mathrm{x}<1$. For $\mathrm{x}<0.4$ the spectra have a typical semiconductor behavior which disappears as the sodium concentration overcomes the threshold value for the IMT transition. 


\section{S5 - Morphology}
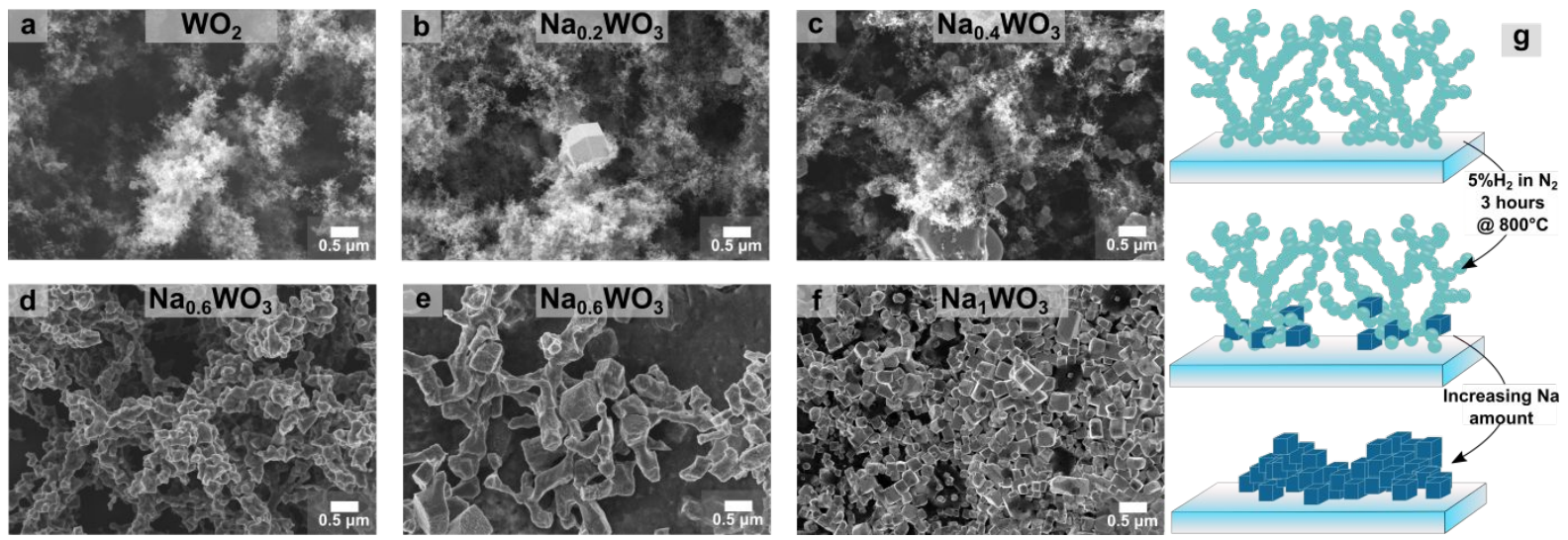

Figure S5. Scanning electron micrograph of the $\mathrm{Na}_{\mathrm{x}} \mathrm{WO}_{3}$, with $0<\mathrm{x}<1$. For sodium concentrations $\mathrm{x} \leq 0.4$ the system presents aggregates of nanoparticles organized in fractal structures showing ultra-high porosity, in line with the diffusion-limited process to which the supersaturated aerosol from the flame spray pyrolysis is subjected ${ }^{4,7}$. Above $\mathrm{x} \sim 0.4$, the high temperature and the reducing atmosphere cause a morphological and structural change which progressively leads to the reduction of the porosity and to the formation of regular denser cubic structures (for $\mathrm{x}=1$ ).
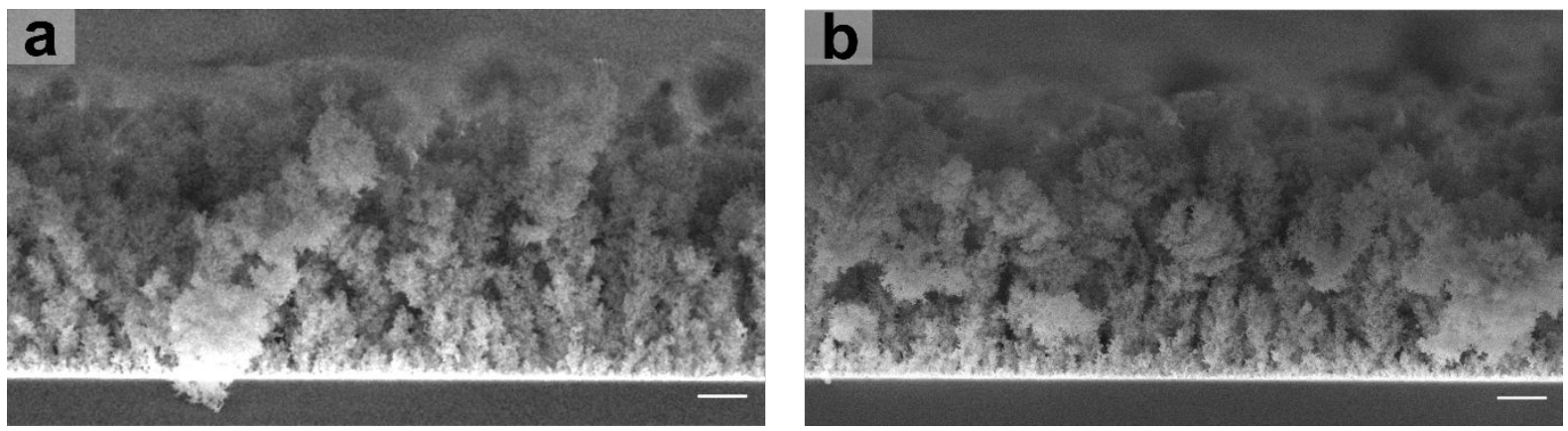

Figure S6. Cross-sectional SEM image of as-deposited $\mathrm{WO}_{3}$ and $\mathrm{Na}_{1} \mathrm{WO}_{3}$ films before the thermal reduction showing a similar average thickness of $\sim 5.1 \mu \mathrm{m}$. Scale bars are $1 \mu \mathrm{m}$. 


\section{Methods}

$\mathrm{Na}_{\mathrm{x}} \mathrm{WO}_{3}$ metamaterials were prepared by a two-steps process consisting of an aerosol technique based on flame spray pyrolysis of liquid precursors followed by thermal reduction. The metal precursor solutions were prepared by dissolving ammonium (meta) tungstate hydrate (Aldrich, purity $>97 \%$ ) in a $1: 1 \mathrm{v} / \mathrm{v}$ mixture of diethylene glycol monobutyl ether (Aldrich, purity $>98.5 \%$ ) and ethanol (Aldrich, purity $>99.5 \%$ ). Sodium acetate trihydrate (Aldrich, purity $>99.0 \%$ ) was added to reach the desired $\mathrm{Na} / \mathrm{W}$ metal ratios form 0 to 1 . The solutions were fed at $5 \mathrm{~mL} \mathrm{~min}{ }^{-1}$ through a custom-built FSP nozzle by a syringe pump and atomized with oxygen flow $\left(5 \mathrm{~L} \mathrm{~min}^{-1}\right)$ at a constant pressure drop of 4 bar. The resulting spray was ignited with a premixed methane/oxygen flame $\left(\mathrm{CH}_{4}\right.$-flamelet $1.8 \mathrm{~L} \mathrm{~min}^{-1}, \mathrm{O}_{2^{-}}$ flamelet $2.0 \mathrm{~L} \mathrm{~min}^{-1}$ ). The $\mathrm{Na}$ doped $\mathrm{WO}_{3}$ nanoparticle networks were directly deposited onto cleaned quartz substrates at a distance from the burner of $20 \mathrm{~cm}$. A sequent thermal reduction in reducing atmosphere $\left(5 \% \mathrm{H}_{2}\right.$ in $\left.\mathrm{N}_{2}\right)$ for 3 hours at $800{ }^{\circ} \mathrm{C}$ led to the $\mathrm{Na}_{\mathrm{x}} \mathrm{WO}_{3}$ metamaterials.

The morphological study and elemental analysis via energy dispersive spectroscopy (EDS) of the samples were carried out by using a Zeiss Ultraplus (FESEM) instrument at $3 \mathrm{kV}$. The crystallinity was characterized by X-ray diffraction using a Bruker instrument (XRD, D2 Phaser, USA) employing $\mathrm{Cu} \mathrm{K \alpha}$ radiation of wavelength $1.54059 \AA$. Transmission electron micrographs were acquired using a high-resolution field emission microscope Jeol 2100F. Electron energy loss spectroscopy (EELS) measurements were acquired using a JEOL JEM ARM 200CF instrument equipped with a cold field emission gun and an ASCOR fifth-order probe corrector.

Extinction measurements were performed using a linear transmittance setup equipped with a UV-Vis broadband halogen lamp (OSL2, Thorlab) that is then delivered to a quartz sensing chamber and collected by a modular modular spectrometer Ocean Optics FlameS-ESX. An optimal focus of the beam was achieved with a series of collimating and objective lenses, as well as adjustable apertures, from ThorLab.

Band structure and density of the states were calculated within the framework of density functional theory (DFT) with the Quantum Espresso Package ${ }^{8}$ under the generalized gradient approximation (GGA) to solve the Kohn-Sham equations. We used plane augmented wave (PAW) with nonlinear core relativistic correction pseudopotentials. A grid of 40x40x40 kpoints was chosen as a mesh for mapping the Brillouin zone.

Finite-difference time-domain (FDTD) simulations performed with a commercially available software (Lumerical FDTD), were used to investigate the electrodynamic behaviour of the ENZ material. Perfectly matched layers were employed as boundary conditions to avoid the wave reflection at the boundary. A total-field scattered-field source $x$-polarized was chosen to excite the system from the backside (substrate) of the structure. A nonuniform meshing refinement was used to guarantee a precise result with a smallest mesh cell of $0.2 \mathrm{~nm}$ to increase the accuracy of the simulation. The optical properties of $\mathrm{Na}_{0.6} \mathrm{WO}_{3}$ were taken from the Owen reference ${ }^{9}$. The system was approximated with a $\mathrm{Na}_{0.6} \mathrm{WO}_{3}$ cube with a $100 \mathrm{~nm}$ edge on a quartz substrate $\left(\mathrm{n}_{\text {subst }}=1.45\right)$. The near-field enhancements and the scattering cross-sections were monitored using a set of frequency domain probes. 


\section{Appendix A}

Analytically, within a classic framework, the optical properties of metallic materials subjected to an external electromagnetic field are described in terms free-electron response by the complex dielectric function, which can be divided in contributions from interband and intraband transition, where the former follows the Fermi's golden rule while the latter is often described by a Drude model:

$$
\begin{aligned}
\varepsilon(\omega)= & \varepsilon_{\text {inter }}(\omega)+\varepsilon_{\text {Drude }}(\omega)=\varepsilon^{\prime}(\omega)+i \varepsilon^{\prime \prime}(\omega) \\
& =\varepsilon_{i b}-\frac{\omega_{p}^{2}}{\left(\omega^{2}+\gamma^{2}\right)}+i \frac{\omega_{p}^{2} \gamma}{\omega\left(\omega^{2}+\gamma^{2}\right)}
\end{aligned}
$$

Here, $\varepsilon_{i b}$ is the background permittivity accounting for interband contribution, $\gamma$ is the damping parameter which takes into account the overall dissipation effects and $\omega_{p}$ is the plasma frequency. This latter is particularly important because it characterizes the speed of electrons (with effective mass $m_{e}$, charge $e$ and density $n_{e}$ ) to collectively react to an external applied electric potential, being $\varepsilon_{0}$ the vacuum permittivity. Mathematically:

$$
\omega_{p}=\left(n_{e} e^{2} / m_{e} \varepsilon_{0}\right)^{\frac{1}{2}}
$$

Typically, the optical losses are significantly high above the onset of the interband transitions, especially in the UV and visible portion of the electromagnetic spectrum, which determines a hard threshold on the operating frequencies of a plasmonic material. ${ }^{10}$ These limitations imposed by the inherent metal losses have driven the plasmonics community to finding novel alternative materials with a smaller but still real part of the dielectric function and considerably smaller losses, (smaller imaginary part). ${ }^{10,11}$

However, despite a vast gamut of plasmonic-enabled applications, ranging from light harvesting with hot-electrons ${ }^{12}$ to optical communication ${ }^{13}$ and transformation optics, ${ }^{14}$ have been demonstrated on a lab-scale, the extension to functional devices is hindered by the high intrinsic losses at visible frequencies of metallic scatterers, which are deeply connected to the imaginary part of the permittivity, $\varepsilon " '{ }^{15}$ In fact, the excitation of free carriers necessarily involves photon absorption which results in interband vertical and 'diagonal' transitions in the Brillouin zone, or, upon additional momentum, intraband non-vertical transitions. ${ }^{16}$ Ultimately, this is followed by thermal dissipation through different channels, such as thermalization via electron-electron scattering, electron-phonon coupling or via thermal radiation and heat conduction. ${ }^{17}$

Additionally, the causality condition forbids the possibility to have lossless and negative permittivity simultaneously for all frequencies in any dispersive material ${ }^{18}$. However, is possible to tailor the dielectric function to achieve pure lossless and negative permittivity within a frequency interval of the electromagnetic spectrum, but generally not in the visible range for non-metal materials.

Despite this is very much suitable and advantageous for IR applications, one would possibility want to be working within the visible region. To this extent, an alternative research direction includes a tailoring of the permittivity to enable different physical effects 
that have the potentiality to achieve the same performances of plasmonic materials for different applications.

\section{Appendix B}

The interband transitions contribute to imaginary part of the permittivity, $\varepsilon$ "', can be modelled by the Fermi's golden rule: ${ }^{19}$

$$
\varepsilon^{\prime \prime}{ }_{\text {inter }}(\omega) \propto \frac{1}{\omega} \sum_{\mathrm{vck}}\left|\vec{\theta}\left\langle\mathrm{v}_{\vec{k}}|\vec{p}| \mathrm{c}_{\vec{k}}\right\rangle\right|^{2} \delta\left(\mathrm{E}_{\mathrm{ck}}-\mathrm{E}_{\mathrm{vk}}-\omega\right)
$$

Where $\theta$ is the light polarization, $\vec{p}$ is the perturbation momentum operator, and the summation is extended to all the occupied valence band states, $|v \vec{k}\rangle$, and the unoccupied conduction states $|c \vec{k}\rangle$.

Instead, the optical losses due to the intraband contribution are well expressed by the Drude model as following:

$$
\varepsilon^{\prime \prime}{ }_{\text {intra }}(\omega)=\frac{\omega_{p}^{2} \gamma}{\omega\left(\omega^{2}+\gamma^{2}\right)}
$$

Where $\gamma$ is the damping parameter and $\omega_{p}$ is the plasma frequency. 


\section{References}

1. Kubrin, R.; Tricoli, A.; Camenzind, A.; Pratsinis, S. E.; Bauhofer, W. Flame aerosol deposition of Y2O3:Eu nanophosphor screens and their photoluminescent performance. Nanotechnology 2010, 21, (22), 225603.

2. Tricoli, A.; Nasiri, N.; Chen, H.; Wallerand, A. S.; Righettoni, M. Ultra-rapid synthesis of highly porous and robust hierarchical $\mathrm{ZnO}$ films for dye sensitized solar cells. Solar Energy 2016, 136, 553-559.

3. Tran-Phu, T.; Chen, H.; Bo, R.; Di Bernardo, I.; Fusco, Z.; Simonov, A. N.; Tricoli, A. HighTemperature One-Step Synthesis of Efficient Nanostructured Bismuth Vanadate Photoanodes for Water Oxidation. Energy Technology 2019, 7, (8), 1801052.

4. Fusco, Z.; Rahmani, M.; Bo, R.; Verre, R.; Motta, N.; Käll, M.; Neshev, D.; Tricoli, A. Nanostructured Dielectric Fractals on Resonant Plasmonic Metasurfaces for Selective and Sensitive Optical Sensing of Volatile Compounds. Advanced Materials 2018, 30, (30), 1800931.

5. $\quad$ Tran-Phu, T.; Daiyan, R.; Fusco, Z.; Ma, Z.; Amal, R.; Tricoli, A. Nanostructured $\beta-B i 2 O 3$ Fractals on Carbon Fibers for Highly Selective $\mathrm{CO} 2$ Electroreduction to Formate. Advanced Functional Materials 2019, n/a, (n/a), 1906478.

6. Raj, S.; Matsui, H.; Souma, S.; Sato, T.; Takahashi, T.; Chakraborty, A.; Sarma, D. D.; Mahadevan, P.; Oishi, S.; McCarroll, W. H.; Greenblatt, M. Electronic structure of sodium tungsten bronzesNaxWO3by high-resolution angle-resolved photoemission spectroscopy. Physical Review B 2007, 75, (15).

7. Tricoli, A.; Elmøe, T. D. Flame spray pyrolysis synthesis and aerosol deposition of nanoparticle films. AlChE Journal 2012, 58, (11), 3578-3588.

8. Giannozzi, P.; Baroni, S.; Bonini, N.; Calandra, M.; Car, R.; Cavazzoni, C.; Ceresoli, D.; Chiarotti, G. L.; Cococcioni, M.; Dabo, I.; Dal Corso, A.; de Gironcoli, S.; Fabris, S.; Fratesi, G.; Gebauer, R.; Gerstmann, U.; Gougoussis, C.; Kokalj, A.; Lazzeri, M.; Martin-Samos, L.; Marzari, N.; Mauri, F.; Mazzarello, R.; Paolini, S.; Pasquarello, A.; Paulatto, L.; Sbraccia, C.; Scandolo, S.; Sclauzero, G.; Seitsonen, A. P.; Smogunov, A.; Umari, P.; Wentzcovitch, R. M. QUANTUM ESPRESSO: a modular and open-source software project for quantum simulations of materials. Journal of Physics: Condensed Matter 2009, 21, (39), 395502.

9. Owen, J. F.; Teegarden, K. J.; Shanks, H. R. Optical properties of the sodium-tungsten bronzes and tungsten trioxide. Physical Review $B$ 1978, 18, (8), 3827-3837.

10. West, P. R.; Ishii, S.; Naik, G. V.; Emani, N. K.; Shalaev, V. M.; Boltasseva, A. Searching for better plasmonic materials. Laser \& Photonics Reviews 2010, 4, (6), 795-808.

11. Naik, G. V.; Shalaev, V. M.; Boltasseva, A. Alternative plasmonic materials: beyond gold and silver. Adv Mater 2013, 25, (24), 3264-94.

12. Zhou, N.; López-Puente, V.; Wang, Q.; Polavarapu, L.; Pastoriza-Santos, I.; Xu, Q.-H. Plasmonenhanced light harvesting: applications in enhanced photocatalysis, photodynamic therapy and photovoltaics. RSC Advances 2015, 5, (37), 29076-29097.

13. Leuthold, J.; Hoessbacher, C.; Muehlbrandt, S.; Melikyan, A.; Kohl, M.; Koos, C.; Freude, W.; Dolores-Calzadilla, V.; Smit, M.; Suarez, I.; Martínez-Pastor, J.; Fitrakis, E. P.; Tomkos, I. Plasmonic Communications: Light on a Wire. Opt. Photon. News 2013, 24, (5), 28-35.

14. Pendry, J. B.; Schurig, D.; Smith, D. R. Controlling Electromagnetic Fields. Science 2006, 312, (5781), 1780-1782.

15. Khurgin, J. B.; Boltasseva, A. Reflecting upon the losses in plasmonics and metamaterials. MRS Bulletin 2012, 37, (8), 768-779. 
16. Gjerding, M. N.; Pandey, M.; Thygesen, K. S. Band structure engineered layered metals for low-loss plasmonics. Nature Communications 2017, 8, 15133.

17. Boriskina, S. V.; Cooper, T. A.; Zeng, L.; Ni, G.; Tong, J. K.; Tsurimaki, Y.; Huang, Y.; Meroueh, L.; Mahan, G.; Chen, G. Losses in plasmonics: from mitigating energy dissipation to embracing loss-enabled functionalities. Adv. Opt. Photon. 2017, 9, (4), 775-827.

18. Dressel, M.; Gruener, G. Electrodynamics of Solids: Optical Properties of Electrons in Matter. American Journal of Physics 2002, 70, (12), 1269-1270.

19. Xue, Y.; Zhang, Y.; Zhang, P. Theory of the color change ofNaxWO3as a function of Na-charge doping. Physical Review B 2009, 79, (20). 